\title{
Exponential bounds for noncommuting systems of matrices
}

\author{
by \\ BRIAN JefFERIES (Sydney, NSW)
}

\begin{abstract}
It is shown that a finite system $T$ of matrices whose real linear combinations have real spectrum satisfies a bound of the form $\left\|e^{i\langle T, \zeta\rangle}\right\| \leq C(1+|\zeta|)^{s} e^{r|\Im \zeta|}$. The proof appeals to the monogenic functional calculus.
\end{abstract}

Introduction. For a system $T=\left(T_{1}, \ldots, T_{d}\right)$ of $n \times n$ matrices with the property that the spectrum $\sigma(\langle T, \xi\rangle)$ of the matrix $\langle T, \xi\rangle:=\sum_{j=1}^{d} T_{j} \xi_{j}$ is real for every $\xi \in \mathbb{R}^{d}$, a symmetric functional calculus for $T$ has been defined in [7]. The matrices $T_{1}, \ldots, T_{d}$ do not necessarily commute with each other. The functional calculus has the property that if $p: \mathbb{R}^{d} \rightarrow \mathbb{C}$ is a polynomial in $d$ real variables, then $p(T)$ is the matrix obtained by replacing the expressions $x_{j_{1}} \ldots x_{j_{k}}$ in $p$ by symmetrised products of the matrices $T_{j_{1}}, \ldots, T_{j_{k}}$.

In the case that $T$ is a $d$-tuple of hermitian matrices, the Weyl functional calculus [12], [10], [1] is also defined for $T$ and it associates the same matrix $p(T)$ with the polynomial $p: \mathbb{R}^{d} \rightarrow \mathbb{C}$. If the assumption that $T$ consists of hermitian matrices is replaced by the assumption that there exist numbers $C>0$ and $r, s \geq 0$ such that

$$
\left\|e^{i\langle T, \zeta\rangle}\right\| \leq C(1+|\zeta|)^{s} e^{r|\Im \zeta|} \quad \text { for all } \zeta \in \mathbb{C}^{d},
$$

then the Paley-Wiener Theorem still guarantees the existence of the Weyl functional calculus. Moreover, it is proved in [6] that the Weyl functional calculus and the functional calculus mentioned above agree on their common domain and the two related notions of "joint spectrum" $\gamma(T)$ for the system $T$ coincide.

It is well known that the bound (1) implies that

$$
\sigma(\langle T, \xi\rangle) \subseteq \mathbb{R} \quad \text { for all } \xi \in \mathbb{R}^{d},
$$

2000 Mathematics Subject Classification: Primary 47A60, 46H30; Secondary 47A25, $30 \mathrm{G} 35$.

Key words and phrases: exponential bound, Clifford algebra, monogenic function. 
even for a system $T$ of bounded linear operators acting on a Banach space $[4$, Theorem 4.5, p. 160]. The purpose of this note is to prove that condition (2) implies the bound (1) for a system of matrices. It is a simple matter to write down an example of a commuting pair $T$ of bounded linear operators acting on $\ell^{2}(\mathbb{N})$ such that condition (2) holds but the bound (1) fails $[7$, Example 2.1].

Although $\langle T, \zeta\rangle$ may be put into its Jordan normal form for each $\zeta \in \mathbb{C}^{d}$ so that the eigenvalues are real if $\zeta \in \mathbb{R}^{d}$, it is not obvious how the imaginary parts of the eigenvalues will grow as $|\Im \zeta| \rightarrow \infty$. A further difficulty is that the similarity transformations $U(\zeta)$ which put $\langle T, \zeta\rangle$ into its Jordan normal form $J(\zeta)$ are only holomorphic in an open set in which $J(\zeta)$ has constant structure [2, Theorem 3, p. 387]. This difficulty is bypassed by appealing to a formula of E. Nelson [10], [5] expressing the exponential $e^{i\langle T, \zeta\rangle}$ in terms of powers of $\langle T, \zeta\rangle$ up to order $n-1$.

In Theorem 2 it is shown that, in the language of A. Pryde [11], a system of matrices satisfying (2) is of Paley-Wiener type $(s, r)$, that is, the bound (1) holds. A system of simultaneously triangularisable matrices with real spectrum satisfies (2), so Theorem 2 is a generalisation of [11, Theorem 4.5].

In order to prove Theorem 2, we use the fact, proved in Theorem 1, that the imaginary part of the spectrum of $A t+i B$ is uniformly bounded for all $t \in \mathbb{R}$ provided that the bounded linear operators $A, B$ have the property that every real linear combination of them has real spectrum. This observation may be of independent interest. The proof appeals to properties of the monogenic functional calculus studied in [7] and quoted in the section on the monogenic calculus.

Another proof that condition (1) follows from condition (2) may be deduced from the theory of the hyperbolic system

$$
\frac{\partial u}{\partial t}+\sum_{j=1}^{d} T_{j} \frac{\partial u}{\partial x_{j}}=0, \quad u(x, 0)=\delta_{0}(x) v, \quad v \in \mathbb{R}^{n},
$$

of partial differential equations. Condition (2) is an expression of Gårding's hyperbolicity condition [8, pp. 149-151], which is equivalent to the statement that the system (3) has a suitable fundamental solution. From Holmgren's uniqueness theorem $[8$, p. 83], we know that the domain of dependence of the distributional solution $u$ of (3) is a cone with finite diameter in $\mathbb{R}^{d+1}$ (see [8, p. 153]), from which the bound (1) follows.

Estimates for the parameters $C$ and $r$ are obtained in the course of the proof of Theorem 2 below. Moreover, parts of the proof have the advantage of generalising to systems of bounded linear operators, provided that the assumption (2) is strengthened. 
Perturbation. Suppose that $A, B$ are $(n \times n)$ matrices and the spectrum $\sigma(A)$ of $A$ is real. If $A$ and $B$ commute, then for each $t \in \mathbb{R}$, the spectrum $\sigma(A t+i B)$ of the matrix $A t+i B$ is contained in the set of complex numbers $\lambda t+i \mu$ with $\lambda \in \sigma(A)$ and $\mu \in \sigma(B)$. It follows that $\sup _{t \in \mathbb{R}}|\Im(\sigma(A t+i B))|<\infty$. However, if $A$ and $B$ do not commute, this bound can fail.

By finite-dimensional perturbation theory [9, Theorem II.5.1], the unordered set of eigenvalues of $A t+i B$ is a continuous function of $t$, so for any $T>0$, the set $\bigcup_{|t| \leq T} \Im(\sigma(A t+i B))$ is a compact subset of $\mathbb{R}$. On the other hand, because $\sigma(\bar{A})$ is real, $\Im(\sigma(A+i B / t)) \rightarrow\{0\}$ as $|t| \rightarrow \infty$, so that $\Im(\sigma(A t+i B))=\{o(|t|)\}$ as $|t| \rightarrow \infty$. The following example shows that if $n \geq 2$, the set $\Im(\sigma(A t+i B))$ may not be bounded as $|t| \rightarrow \infty$.

ExAmple. Let $A=\left(\begin{array}{ll}1 & 1 \\ 0 & 1\end{array}\right)$ and $B=\left(\begin{array}{ll}1 & 0 \\ 1 & 1\end{array}\right)$. Then $\sigma(A t+i B)=\{t+i \pm$ $\left.e^{i \pi / 4} \sqrt{t}\right\}$ for all $t \geq 0$. On the other hand, if $B^{\prime}=\left(\begin{array}{cc}1 & 1 \\ 0 & -1\end{array}\right)$, then $\sigma\left(A t+i B^{\prime}\right)=$ $\{t \pm i\}$.

The matrices $A$ and $B^{\prime}$ above are both upper triangular and each real linear combination of them has real spectrum. By contrast, the matrices $A$ and $B$ each have real spectrum but $\sigma(A-B)=\{ \pm i\}$. Moreover,

$$
e^{i(A-B) t}=\cosh (t) I+\sinh (t)\left(\begin{array}{cc}
0 & i \\
-i & 0
\end{array}\right),
$$

so the bound (1) certainly fails.

To prove Theorem 2 we only need the following result for matrices, but the proof of Theorem 1 is also valid for bounded linear operators acting on a Banach space.

THEOREM 1. Let $A, B$ be bounded linear operators acting on the Banach space $X$ with the property that $\sigma\left(A \xi_{1}+B \xi_{2}\right) \subset \mathbb{R}$ for all $\xi \in \mathbb{R}^{2}$. Then there exist $q, r>0$ such that

$$
\sigma(A t+i B) \subseteq[-q, q] t+i[-r, r] \quad \text { for all } t \in \mathbb{R} .
$$

Moreover, $q$ and $r$ are bounded by $(1+\sqrt{2})\left(\|A\|^{2}+\|B\|^{2}\right)^{1 / 2}$.

Monogenic functional calculus. In order to bound the imaginary part of the spectrum $\sigma(A t+i B)$ for large $t$, we need to determine for which $\lambda \in \mathbb{C}$ the resolvent operator $(\lambda I-A t-i B)^{-1}$ of $A t+i B$ is defined as $t$ varies. To this end, the resolvent operator $(\lambda I-A t-i B)^{-1}$ is represented as a function $f_{\lambda, t}(A, B)$ of the pair of operators $A, B$ with $f_{\lambda, t}\left(x_{1}, x_{2}\right)=$ $\left(\lambda I-x_{1} t-i x_{2}\right)^{-1}$.

Now the operators $A$ and $B$ do not necessarily commute, so it is not yet clear how to form functions $f(A, B)$ of $A$ and $B$. If an estimate like (1) held for the pair $T=(A, B)$, then the Weyl functional calculus $\mathcal{W}_{T}$ would exist 
and the equality

$$
(\lambda I-A t-i B)^{-1}=\mathcal{W}_{T}\left(f_{\lambda, t}\right)
$$

would hold for all $\lambda \notin\left\{x_{1} t+i x_{2}:\left(x_{1}, x_{2}\right) \in \operatorname{supp}\left(\mathcal{W}_{T}\right)\right\}$. However, we are trying to deduce the exponential estimate (1) for the case that $A$ and $B$ are matrices. What we need is a functional calculus $f \mapsto f(A, B)$ valid just under the assumption that $\sigma\left(A \xi_{1}+B \xi_{2}\right) \subset \mathbb{R}$ for all $\xi \in \mathbb{R}^{2}$ so that

$$
f_{\lambda, t}(A, B)=(\lambda I-A t-i B)^{-1} .
$$

The monogenic functional calculus developed in [7] is useful in this context. Its description follows.

The notation of [3] concerning Clifford algebras is used. If $\mathbb{F}$ denotes the field $\mathbb{R}$ or $\mathbb{C}$, then $\mathbb{F}_{(m)}$ denotes the Clifford algebra over $\mathbb{F}$ generated by $e_{0}, e_{1}, \ldots, e_{m}$. For a Banach space $X$, the family of sums $T=\sum_{S} T_{S} e_{S}$ for $T_{S} \in \mathcal{L}(X)$ and $S \subseteq\{1, \ldots, m\}$ forms a Banach module $\mathcal{L}_{(m)}\left(X_{(m)}\right)$ under left and right multiplication by elements of $\mathbb{F}_{(m)}$. The norm is given by $\|T\|=\left(\sum_{S}\left\|T_{S}\right\|_{\mathcal{L}(X)}^{2}\right)^{1 / 2}$.

Let $D$ be the differential operator $D=\sum_{j=0}^{m} e_{j} \partial / \partial x_{j}$. A function $f$ : $U \rightarrow \mathbb{F}_{(m)}$ is called left monogenic in an open set $U$ if $D f=0$ in $U$. It is right monogenic in $U$ if $f D=0$ in $U$. The expression two-sided monogenic is used for functions which are both left and right monogenic. For each $\omega \in \mathbb{R}^{m+1}$, the function $G_{\omega}$ defined by

$$
G_{\omega}(x)=\frac{1}{\sigma_{m}} \frac{\overline{\omega-x}}{|\omega-x|^{m+1}} \quad \text { for each } x \neq \omega
$$

is two-sided monogenic. Here $\sigma_{m}=2 \pi^{(m+1) / 2} / \Gamma((m+1) / 2)$ is the volume of the unit $m$-sphere in $\mathbb{R}^{m+1}$ and $\mathbb{R}^{m+1}$ is itself identified with a linear subspace of $\mathbb{R}_{(m)}$ spanned by the basis vectors $e_{0}, e_{1}, \ldots, e_{m}$. The notation $E(\omega-x)=G_{\omega}(x)$ is used in [3].

Suppose that $\Omega \subset \mathbb{R}^{m+1}$ is a bounded open set with smooth boundary $\partial \Omega$ and exterior unit normal $n(\omega)$ defined for all $\omega \in \partial \Omega$. For any left monogenic function $f$ defined in a neighbourhood $U$ of $\bar{\Omega}$, the Cauchy integral formula

$$
\int_{\partial \Omega} G_{\omega}(x) n(\omega) f(\omega) d \mu(\omega)= \begin{cases}f(x) & \text { if } x \in \Omega, \\ 0 & \text { if } x \in U \backslash \bar{\Omega},\end{cases}
$$

is valid. Here $\mu$ is the surface measure of $\partial \Omega$. The result is proved in [3, Corollary 9.6]. If $g$ is right monogenic in $U$ then $\int_{\partial \Omega} g(\omega) n(\omega) f(\omega) d \mu(\omega)=0$ (see [3, Corollary 9.3]).

These results extend to the vector- and operator-valued setting in a routine fashion. In this case, "monogenic" means that the partial derivatives are evaluated in the underlying topology of the space. 
In the monogenic functional calculus for a suitable $m$-tuple $A$ of bounded operators acting on a Banach space $X$, the operator $f(A)$ is defined for all $\mathbb{F}$-valued functions $f$ of $m$-real variables analytic in an open neighbourhood $U$ of the monogenic spectrum $\gamma(A)$ (see [7, Section 3]). The operator $f(A)$ is defined analogously to the Cauchy integral formula (6), where $G_{\omega}$ is replaced by a suitable element $G_{\omega}(A)$ of $\mathcal{L}_{(m)}\left(X_{(m)}\right)$ for each $\omega \in \mathbb{R}^{m+1} \backslash \gamma(A)$ and $f$ is extended monogenically off $\{0\} \times U$ into $\mathbb{R}^{m+1}$.

In [7], the Cauchy kernel $\omega \mapsto G_{\omega}(A), \omega \in \mathbb{R}^{m+1} \backslash \gamma(A)$, is identified by employing he plane wave decomposition of the Cauchy kernel (5).

According to [7], there exists a compact subset $\gamma(A, B)$ of $\mathbb{R}^{2}$ and an $\mathcal{L}_{(2)}\left(X_{(2)}\right)$-valued monogenic function $\omega \mapsto G_{\omega}(A, B)$ defined for all $\omega \in \mathbb{R}^{3}$ off $\{0\} \times \gamma(A, B)$ such that

$$
f(A, B)=\int_{\partial \Omega} G_{\omega}(A, B) n(\omega) f(\omega) d \mu(\omega) .
$$

Here $\Omega \subset \mathbb{R}^{3}$ is a bounded open set with smooth boundary $\partial \Omega$ and exterior unit normal $n(\omega)$ defined for all $\omega \in \partial \Omega$ such that $\Omega$ contains $\{0\} \times \gamma(A, B)$. The surface measure of $\partial \Omega$ is $\mu$ and $f$ is a $\mathbb{C}_{(n)}$-valued function that is monogenic in a neighbourhood of $\bar{\Omega}$. According to [7, Lemma 2.5], the formula for $G_{\omega}(A, B)$ for $\omega$ off $\{0\} \times \mathbb{R}^{2}$ is

$$
\begin{array}{r}
G_{y+y_{0} e_{0}}(A, B)=-\frac{\operatorname{sgn}\left(y_{0}\right)}{8 \pi^{2}} \int_{S_{1}}\left(\langle y, s\rangle I-A s_{1}-B s_{2}-y_{0} s I\right)^{-2} d \mu(s), \\
y \in \mathbb{R}^{2}, y_{0} \neq 0 .
\end{array}
$$

Here $\mu$ is arclength measure on the unit circle $S_{1}$ in $\mathbb{R}^{2}$ and the inverse $\left(\langle y, s\rangle I-A s_{1}-B s_{2}-y_{0} s I\right)^{-2}$ is calculated in the Banach module $\mathcal{L}_{(2)}\left(X_{(2)}\right)$.

In the case that $f$ is a complex-valued analytic function of two real variables in an open neighbourhood of the compact subset $\gamma(A, B)$ of $\mathbb{R}^{2}$, we obtain an operator $f(A, B) \in \mathcal{L}(X)$ from formula $(7)$ by extending $f$ monogenically to an open neighbourhood of $\{0\} \times \gamma(A, B)$ in $\mathbb{R}^{3}$ (see $[7$, Theorem 3.5(iv)]).

The nonempty compact subset $\gamma(A, B)$ of $\mathbb{R}^{2}$ is the monogenic spectrum of the pair $(A, B)$. In the case that an exponential bound like (1) holds, $\gamma(A, B)$ coincides with the support of the Weyl functional calculus for $(A, B)$ (see [6]). As is usual in spectral theory, $\gamma(A, B)$ is just the set of singularities of the Cauchy kernel $\omega \mapsto G_{\omega}(A, B)$ in the same way that the spectrum of a single operator is the set of singularities of its resolvent family.

Once we have the notion of the monogenic functional calculus, the proof of the following lemma is straightforward.

Lemma 1. Let $A, B$ be bounded linear operators acting on the Banach space $X$ with the property that $\sigma\left(A \xi_{1}+B \xi_{2}\right) \subset \mathbb{R}$ for all $\xi \in \mathbb{R}^{2}$. Let $\gamma(A, B)$ 
be the monogenic spectrum of the pair $(A, B)$. For every $\lambda \in \mathbb{C}$ and $t \in \mathbb{R}$, set

$$
f_{\lambda, t}(x)=\left(\lambda-x_{1} t-i x_{2}\right)^{-1}
$$

for all $x=\left(x_{1}, x_{2}\right) \in \mathbb{R}^{2}$ such that $x_{1} t+i x_{2} \neq \lambda$. Then for every $t \in \mathbb{R}$, the complement $R_{t}(A, B)$ of the set $\left\{x_{1} t+i x_{2}:\left(x_{1}, x_{2}\right) \in \gamma(A, B)\right\}$ is contained in the resolvent set of the operator $A t+i B$ and the equality

$$
f_{\lambda, t}(A, B)=(\lambda I-A t-i B)^{-1}, \quad \lambda \in R_{t}(A, B),
$$

is valid.

Proof. For each $j=1,2$, the unique left and right monogenic extension of the coordinate function $x \mapsto x_{j}, x \in \mathbb{R}^{2}$, is $\omega \mapsto \omega_{j} e_{0}-\omega_{0} e_{j}, \omega=$ $\left(\omega_{0}, \omega_{1}, \omega_{2}\right) \in \mathbb{R}^{3}$.

Let $\widetilde{f}_{\lambda, t}$ be the $\mathbb{C}_{(2)}$-valued function given by

$$
\begin{aligned}
\tilde{f}_{\lambda, t}(\omega) & =\left(\lambda e_{0}-\left(\omega_{1} e_{0}-\omega_{0} e_{1}\right) t-i\left(\omega_{2} e_{0}-\omega_{0} e_{2}\right)\right)^{-1} \\
& =\frac{\left(\bar{\lambda}-\omega_{1} t+i \omega_{2}\right) e_{0}-\omega_{0} t e_{1}+i \omega_{0} e_{2}}{\left|\lambda-\omega_{1} t-i \omega_{2}\right|^{2}+\omega_{0}^{2}\left(t^{2}+1\right)}
\end{aligned}
$$

for all $\omega \in \mathbb{R}^{3}$ for which the denominator is nonzero. The restriction of $\widetilde{f}_{\lambda, t}$ to $\mathbb{R}^{2}$ is equal to $f_{\lambda, t}$, that is, on putting $\omega_{0}=0$. The function $\widetilde{f}_{\lambda, t}$ takes its values in the linear subspace spanned by $e_{0}, e_{1}, e_{2}$ in $\mathbb{C}_{(2)}$ and is left and right monogenic. Then for every complex number $\lambda \in R_{t}(A, B)$, the operator $\widetilde{f}_{\lambda, t}(A, B)$ is defined by formula (7) for a suitable choice of the open set $\Omega$.

The Neumann series expansion

$$
\sum_{k=0}^{\infty} \frac{1}{\lambda^{k+1}}(A t+i B)^{k}
$$

of $(\lambda I-A t-i B)^{-1}$ converges for $|\lambda|$ large enough. Moreover, the sums

$$
\begin{aligned}
& f_{\lambda, t}(x)=\sum_{k=0}^{\infty} \frac{1}{\lambda^{k+1}}\left(x_{1} t+i x_{2}\right)^{k} \\
& \widetilde{f}_{\lambda, t}(\omega)=\sum_{k=0}^{\infty} \frac{1}{\lambda^{k+1}}\left(\left(\omega_{1} e_{0}-\omega_{0} e_{1}\right) t+i\left(\omega_{2} e_{0}-\omega_{0} e_{2}\right)\right)^{k}
\end{aligned}
$$

converge uniformly as $x \in \mathbb{R}^{2}$ and $\omega \in \mathbb{R}^{3}$ range over compact sets and $\lambda$ is outside a sufficiently large ball. Set

$$
\begin{aligned}
& p_{k, t}(x)=\left(x_{1} t+i x_{2}\right)^{k}, \quad x \in \mathbb{R}^{2}, \\
& \widetilde{p}_{k, t}(\omega)=\left(\left(\omega_{1} e_{0}-\omega_{0} e_{1}\right) t+i\left(\omega_{2} e_{0}-\omega_{0} e_{2}\right)\right)^{k}, \quad \omega \in \mathbb{R}^{3},
\end{aligned}
$$

for each $k=0,1,2, \ldots$ 
Now $f_{\lambda, t}(A, B)=\widetilde{f}_{\lambda, t}(A, B)$, by definition, for all $\lambda \in R_{t}(A, B)$. According to $[7$, Theorem $3.5($ ii) $]$, the equality

$$
p_{k, t}(A, B)=\widetilde{p}_{k, t}(A, B)=(A t+i B)^{k}
$$

holds for all $k=0,1,2 \ldots$, so by the continuity of the monogenic functional calculus $f \mapsto f(A, B)$ (see [7, Proposition 3.3]), we have

$$
f_{\lambda, t}(A, B)=\sum_{k=0}^{\infty} \frac{1}{\lambda^{k+1}}(A t+i B)^{k}=(\lambda I-A t-i B)^{-1},
$$

that is,

$$
(\lambda I-A t-i B) f_{\lambda, t}(A, B)=f_{\lambda, t}(A, B)(\lambda I-A t-i B)=I
$$

for all $\lambda \in \mathbb{C}$ with $|\lambda|$ sufficiently large.

Now $f_{\lambda, t}(A, B)$ is defined by formula (7) for all $\lambda \in R_{t}(A, B)$, and by differentiation under the integral (7), we see that $\lambda \mapsto f_{\lambda, t}(A, B), \lambda \in R_{t}(A, B)$, is a complex-analytic $\mathcal{L}(X)$-valued function. It follows that equation (9) holds for all $\lambda \in R_{t}(A, B)$, so that the resolvent set of the operator $A t+i B$ contains the $R_{t}(A, B)$ and the equality (8) holds.

Proof of Theorem 1. Put

$$
\begin{aligned}
& q=\sup \left\{\left|x_{1}\right|:\left(x_{1}, x_{2}\right) \in \gamma(A, B)\right\}, \\
& r=\sup \left\{\left|x_{2}\right|:\left(x_{1}, x_{2}\right) \in \gamma(A, B)\right\} .
\end{aligned}
$$

Then for every $t \in \mathbb{R}$, the complement of the rectangle $[-q, q] t+i[-r, r]$ is contained in the set $R_{t}(A, B)$ defined in Lemma 1 . The inclusion (4) follows from Lemma 1.

The bound for $q, r$ follows from the expansion for $G_{\omega}(A, B)$ which converges for $|\omega|>(1+\sqrt{2})\left(\|A\|^{2}+\|B\|^{2}\right)^{1 / 2}$ (see [7, equation (5)]).

REMARK. For bounded selfadjoint operators $A, B$ acting on a Hilbert space, the spectrum $\sigma(A t+i B)$ is contained in the numerical range of $A t+i B$, so the result follows immediately.

The bound. This section is devoted to proving the following result, in which an algebraic condition implies a matrix-norm bound on exponentials. Let $n \geq 2$ be an integer.

TheOREM 2. Let $T=\left(T_{1}, \ldots, T_{d}\right)$ be a d-tuple of $n \times n$ matrices such that

$$
\sigma(\langle T, \xi\rangle) \subseteq \mathbb{R} \quad \text { for all } \xi \in \mathbb{R}^{d} .
$$

Then there exist numbers $C>0$ and $r \geq 0$ such that

$$
\left\|e^{i\langle T, \zeta\rangle}\right\| \leq C(1+|\zeta|)^{n-1} e^{r|\Im \zeta|} \quad \text { for all } \zeta \in \mathbb{C}^{d} .
$$


We first observe that for all $\zeta \in \mathbb{C}^{d}$ satisfying $|\Re \zeta| \leq 1$, the bound

$$
\left\|e^{i\langle T, \zeta\rangle}\right\| \leq e^{R} e^{R|\Im \zeta|}
$$

holds for $R=\left(\sum_{j=1}^{d}\left\|T_{j}\right\|^{2}\right)^{1 / 2}$, so we need only consider the case $|\Re \zeta| \geq 1$.

For any $n \times n$ matrix $M$, and $k=1, \ldots, n$, let $\phi_{k}(M)$ be the sum of the principal minors of $M$ of order $k$ and set $\phi_{0}(M)=1$. The characteristic polynomial $p_{M}$ of $M$ is defined by $p_{M}(z)=\operatorname{det}(M-z I)$ for all $z \in \mathbb{C}$. For each $n \times n$ matrix $M$, let the complex numbers $a_{0}(M), \ldots, a_{n}(M)$ be the coefficients of the characteristic polynomial $p_{M}$ of $M$. The coefficients of the characteristic polynomial of an $n \times n$ matrix $M$ are calculated from the sums of the principal minors by virtue of the equality $a_{s}(M)=(-1)^{s} \phi_{n-s}(M)$, for all $s=0, \ldots, n-1$. In particular, $a_{s}(t M)=t^{n-s} a_{s}(M)$ for each $t>0$ and $s=0, \ldots, n$.

The equality

$$
p_{M}(z) I=(z I-M) \sum_{k=0}^{n-1}\left(\sum_{j=0}^{n-k-1} a_{j+k+1}(M) z^{j}\right) M^{k}
$$

is easily verified from the Cayley-Hamilton theorem.

Now suppose that $C$ is a simple closed curved in $\mathbb{C}$ surrounding the set $\sigma(M)$ of eigenvalues of the $n \times n$ matrix $M$. Then by the Cauchy-Riesz functional calculus,

$$
\begin{aligned}
e^{i M} & =\frac{1}{2 \pi i} \int_{C} e^{i z}(z I-M)^{-1} d z \\
& =\frac{1}{2 \pi i} \sum_{k=0}^{n-1}\left(\sum_{j=0}^{n-k-1} a_{j+k+1}(M) \int_{C} \frac{e^{i z} z^{j}}{p_{M}(z)} d z\right) M^{k} .
\end{aligned}
$$

Let $T$ be a $d$-tuple of $(n \times n)$ matrices such that $\sigma(\langle T, \xi\rangle)$ is real for each $\xi \in \mathbb{R}^{d}$. On setting $M=\langle T, \zeta\rangle$ with $\zeta \in \mathbb{C}^{d}$ in equation (10), we have

$$
e^{i\langle T, \zeta\rangle}=\frac{1}{2 \pi i} \sum_{k=0}^{n-1}\left(\sum_{j=0}^{n-k-1} a_{j+k+1}(\langle T, \zeta\rangle) \int_{C} \frac{e^{i z} z^{j}}{p_{\langle T, \zeta\rangle}(z)} d z\right)\langle T, \zeta\rangle^{k} .
$$

Here $C$ is any simple closed contour with the finite set $\sigma(\langle T, \zeta\rangle)$ in its interior.

Let $S^{(d-1)}=\left\{\zeta \in \mathbb{C}^{d}:|\zeta|=1\right\}$ and $u, v \geq 0$. It follows from [9, Theorem 5.14] and the compactness of $S^{(d-1)}$ in $\mathbb{C}^{d}$ that

$$
\bigcup\left\{\sigma(\langle T, \Re \zeta\rangle u+i\langle T, \Im \zeta\rangle v): \zeta \in S^{(d-1)}\right\}
$$

is a compact subset of $\mathbb{C}$.

According to Theorem 1, there exists $r>0$ such that for every $\xi, \eta \in \mathbb{R}^{d}$ with $\xi+i \eta \in S^{(d-1)}$ and $t>0$, the spectrum $\sigma(\langle T, \xi\rangle t+i\langle T, \eta\rangle)$ of the 
matrix $\langle T, \xi\rangle t+i\langle T, \eta\rangle$ is contained in $t[-r, r]+i[-r, r]$. The number

$$
r=\sup \left\{|x|: x \in \gamma(\langle T, \xi\rangle,\langle T, \eta\rangle), \xi+i \eta \in S^{(d-1)}\right\}
$$

is bounded by $(1+\sqrt{2})\left(\sum_{j=1}^{d}\left\|T_{j}\right\|^{2}\right)^{1 / 2}$.

If $u, v>0$, then setting $t=u / v$, we find that the spectrum of $\langle T, \xi\rangle u+$ $i\langle T, \eta\rangle v$ is contained in $u[-r, r]+i v[-r, r]$. Hence, for every $u, v>0$, the set (11) is contained in the rectangle $u[-r, r]+i v[-r, r]$.

Fix $\xi, \eta \in \mathbb{R}^{d}$ with $\xi+i \eta \in S^{(d-1)}$ and set $A=\langle T, \xi\rangle$ and $B=\langle T, \eta\rangle$. Let $r^{\prime}>r$ and let $C[u, v]$ be the positively oriented closed contour bounding the rectangle $u\left[-r^{\prime}, r^{\prime}\right]+i(1+v)\left[-r^{\prime}, r^{\prime}\right]$. Then for every $u, v \geq 0$, the contour $C[u, v]$ contains the spectrum $\sigma(A u+i B v)$ in its interior and it is bounded away from the $x$-axis as $v \rightarrow 0+$.

Furthermore, the bound

$$
\begin{aligned}
& \left|\int_{C[u, v]} \frac{e^{i z} z^{j}}{p_{A u+i B v}(z)} d z\right| \\
& \leq e^{r^{\prime}} e^{v r^{\prime}}\left(\left(r^{\prime} u\right)^{2}+\left(r^{\prime}(1+v)\right)^{2}\right)^{j / 2} \int_{C[u, v]} \frac{|d z|}{\left|p_{A u+i B v}(z)\right|} \\
& \leq e^{r^{\prime}} e^{v r^{\prime}}\left(r^{\prime}\right)^{j}\left(1+\sqrt{u^{2}+v^{2}}\right)^{j} \int_{C[u, v]} \frac{|d z|}{\left|p_{A u+i B v}(z)\right|}
\end{aligned}
$$

holds. Arclength measure is denoted by $|d z|$. All the roots of the polynomial $p_{A u+i B v}$ are contained in the rectangle $u[-r, r]+i v[-r, r]$.

For all $t>0$ and $0 \leq w \leq 1$, the function $x \mapsto p_{A t+i B w}\left(-x+i r^{\prime}\right)$ is a polynomial of degree $n \geq 2$ whose modulus is bounded below by $\left(r^{\prime}-w r\right)^{n}$, so for all $u \geq 0$ and $v \geq 0$, the upper part of the integral (12) about $C[u, v]$ is bounded by

$$
\begin{aligned}
\int_{-r^{\prime} u}^{r^{\prime} u} \frac{d x}{\left|p_{A u+i B v}\left(-x+i(1+v) r^{\prime}\right)\right|} & \leq \int_{-\infty}^{\infty} \frac{d x}{\left|p_{A u+i B v}\left(-x+i(1+v) r^{\prime}\right)\right|} \\
& =\frac{1}{(1+v)^{n-1}} \int_{-\infty}^{\infty} \frac{d x}{\left|p_{A u^{\prime}+i B v^{\prime}}\left(-x+i r^{\prime}\right)\right|}, \\
& \leq \sup _{t>0,0 \leq w \leq 1} \int_{-\infty}^{\infty} \frac{d x}{\left|p_{A t+i B w}\left(-x+i r^{\prime}\right)\right|} .
\end{aligned}
$$

To see that the supremum is finite, for each $t>0$ and $0 \leq w \leq 1$, let $\Gamma(t, w)$ be the unordered $n$-tuple of eigenvalues of the $(n \times n)$ matrix $A t+i B w$, counting the possible multiplicity of eigenvalues. Then $|\Re \lambda| \leq r t$ and $|\Im \lambda| \leq r$ for each element $\lambda \in \Gamma(t, w)$. 

that

Because $\left|p_{A t+i B w}(z)\right|=\prod_{\lambda \in \Gamma(t, w)}|z-\lambda|$, Hölder's inequality implies

$$
\begin{aligned}
\int_{-\infty}^{\infty} \frac{d x}{\left|p_{A t+i B w}\left(-x+i r^{\prime}\right)\right|} & \leq \prod_{\lambda \in \Gamma(t, w)}\left(\int_{-\infty}^{\infty} \frac{d x}{\left|-x+i r^{\prime}-\lambda\right|^{n}}\right)^{1 / n} \\
& =\prod_{\lambda \in \Gamma(t, w)}\left(\int_{-\infty}^{\infty} \frac{d x}{\left(x^{2}+\left(r^{\prime}-\Im \lambda\right)^{2}\right)^{n / 2}}\right)^{1 / n} \\
& \leq \int_{-\infty}^{\infty} \frac{d x}{\left(x^{2}+\left(r^{\prime}-r\right)^{2}\right)^{n / 2}}=\frac{\sqrt{\pi} \Gamma((n-1) / 2)}{\left(r^{\prime}-r\right)^{n-1} \Gamma(n / 2)}
\end{aligned}
$$

For all $t>0$, the function $y \mapsto p_{A+i B t}\left(r^{\prime}+i y\right), y \in \mathbb{R}$, is a polynomial of degree $n$ whose modulus is bounded below by $\left(r^{\prime}-r\right)^{n}$, so an argument similar to that above shows that for all $u \geq 1$, the right hand part of the integral (12) is bounded by

$$
\begin{aligned}
\int_{-r^{\prime}(1+v)}^{r^{\prime}(1+v)} \frac{d y}{\left|p_{A u+i B v}\left(u r^{\prime}+i y\right)\right|} & \leq \int_{-\infty}^{\infty} \frac{d y}{\left|p_{A u+i B v}\left(u r^{\prime}+i y\right)\right|} \\
& \leq \sup _{t>0} \int_{-\infty}^{\infty} \frac{d y}{\left|p_{A+i B t}\left(r^{\prime}+i y\right)\right|}
\end{aligned}
$$

Let $N_{r^{\prime}}(\xi+i \eta)$ be the maximum of the numbers

$$
\sup _{t>0,0 \leq w \leq 1} \int_{-\infty}^{\infty} \frac{d x}{\left|p_{A t+i B w}\left( \pm\left(-x+i r^{\prime}\right)\right)\right|}, \quad \sup _{t>0} \int_{-\infty}^{\infty} \frac{d y}{\left|p_{A+i B t}\left( \pm\left(r^{\prime}+i y\right)\right)\right|} .
$$

For $\zeta \in \mathbb{C}^{n}$ with $|\Re \zeta| \geq 1$, let $\zeta^{\prime}=\zeta /|\zeta|$. Then

$$
\begin{aligned}
\left\|e^{i\langle T, \zeta\rangle}\right\| \leq & \frac{1}{2 \pi} \sum_{k=0}^{n-1}\left|\left\langle T, \zeta^{\prime}\right\rangle\right|^{k} \sum_{j=0}^{n-k-1}\left|a_{j+k+1}\left(\left\langle T, \zeta^{\prime}\right\rangle\right)\right|\left|\int_{C} \frac{e^{i z} z^{j}}{p_{\langle T, \zeta\rangle}(z)} d z\right||\zeta|^{n-j-1} \\
\leq & \frac{N_{r^{\prime}}\left(\zeta^{\prime}\right) e^{r^{\prime}} e^{r^{\prime}|\Im \zeta|}}{2 \pi} \\
& \times\left.\sum_{k=0}^{n-1}\left|\left\langle T, \zeta^{\prime}\right\rangle\right|^{k} \sum_{j=0}^{n-k-1}\left|a_{j+k+1}\left(\left\langle T, \zeta^{\prime}\right\rangle\right)\right|\left(r^{\prime}\right)^{j}(1+|\zeta|)^{j}|\cdot| \zeta\right|^{n-j-1} \\
\leq & \frac{N_{r^{\prime}}\left(\zeta^{\prime}\right) e^{r^{\prime}}}{2 \pi}\left(\sum_{k=0}^{n-1}\left|\left\langle T, \zeta^{\prime}\right\rangle\right|^{k} \sum_{j=0}^{n-k-1}\left|a_{j+k+1}\left(\left\langle T, \zeta^{\prime}\right\rangle\right)\right|\left(r^{\prime}\right)^{j}\right) \\
& \times(1+|\zeta|)^{n-1} e^{r^{\prime}|\Im \zeta|} \\
\leq & C_{r^{\prime}}(1+|\zeta|)^{n-1} e^{r^{\prime}|\Im \zeta|} .
\end{aligned}
$$


The constant $C_{r^{\prime}}$ is the supremum of

$$
\frac{N_{r^{\prime}}\left(\zeta^{\prime}\right) e^{r^{\prime}}}{2 \pi}\left(\sum_{k=0}^{n-1}\left|\left\langle T, \zeta^{\prime}\right\rangle\right|^{k} \sum_{j=0}^{n-k-1}\left|a_{j+k+1}\left(\left\langle T, \zeta^{\prime}\right\rangle\right)\right|\left(r^{\prime}\right)^{j}\right)
$$

for $\zeta^{\prime} \in S^{(d-1)}$.

\section{References}

[1] R. F. V. Anderson, The Weyl functional calculus, J. Funct. Anal. 4 (1969), 240-267.

[2] H. Baumgärtel, Analytic Perturbation Theory for Matrices and Operators, Oper. Theory Adv. Appl. 15, Birkhäuser, Basel, 1985.

[3] F. Brackx, R. Delanghe and F. Sommen, Clifford Analysis, Res. Notes Math. 76, Pitman, Boston, 1982.

[4] I. Colojoară and C. Foiaş, Theory of Generalized Spectral Operators, Math. Appl. 9, Gordon and Breach, New York, 1968.

[5] B. Jefferies, The Weyl calculus for hermitian matrices, Proc. Amer. Math. Soc. 124 (1996), 121-128.

[6] B. Jefferies and A. McIntosh, The Weyl calculus and Clifford analysis, Bull. Austral. Math. Soc. 57 (1998), 329-341.

[7] B. Jefferies, A. McIntosh and J. Picton-Warlow, The monogenic functional calculus, Studia Math. 136 (1999), 99-119.

[8] F. John, Partial Differential Equations, Springer, Berlin, 4th ed., 1982.

[9] T. Kato, Perturbation Theory for Linear Operators, Springer, Berlin, 2nd ed., 1980.

[10] E. Nelson, Operants: A functional calculus for non-commuting operators, in Functional Analysis and Related Fields, Proc. Conf. in honour of Professor Marshal Stone (Univ. of Chicago, May 1968), F. E. Browder (ed.), Springer, Verlag, Berlin, 1970, 172-187.

[11] A. J. Pryde, Inequalities for exponentials in Banach algebras, Studia Math 100 (1991), 87-94.

[12] M. E. Taylor, Functions of several self-adjoint operators, Proc. Amer. Math. Soc. 19 (1968), 91-98.

School of Mathematics

The University of New South Wales

Sydney, NSW 2052, Australia

E-mail: B.Jefferies@unsw.edu.au

Received June 15, 1998

Revised version January 3, 2001 